\title{
Self hypnotherapeutic treatment of habitual reflex vomiting
}

\author{
B S Sokel, S P Devane, A Bentovim, P J Milla
}

\begin{abstract}
A 9 year old boy with intractable postprandial reflex vomiting was taught a self hypnotherapy technique incorporating relaxation exercises, mental imagery, and suggestions of symptom relief. The sequence was recorded on a personal stereo cassette tape. Vomiting was completely eliminated within four weeks. At 12 month review vomiting had not recurred.
\end{abstract}

Persistent or recurrent vomiting in childhood suggests an underlying serious organic illness. In a small percentage of patients, where no organic cause can be found, the term psychogenic vomiting has been applied and the problem related to factors such as stress avoidance, secondary gain from illness behaviour, anxiety or depression. ${ }^{1}$ Behavioural techniques that modify social contingencies, such as extinction of behaviour by withdrawal of attention and social contact, shaping of behaviour by positive reinforcement of food retention, and 'time-out' for vomiting have been used to treat this group. These are alternatives to the aversive therapy techniques sometimes used with developmentally delayed children, such as mild electric shock treatment, forced vomiting, and aversive taste administration. ${ }^{2}$

Hypnotherapy has been used widely in the treatment of other habit disorders in children. ${ }^{34}$ It has also been useful in the reduction of vomiting associated with the treatment of childhood malignancies. ${ }^{5}$ Apart from a description of the use of hypnosis in two patients referred with cyclic vomiting where an organic explanation was subquently found, ${ }^{6}$ we have been unable to find any reports of the application of hypnotherapy to the treatment of vomiting of no discernible organic cause.

Hospital for

Sick Children,

London,

Department of

Psychological Medicine B S Sokel

A Bentovim

Department of

Child Health,

Institute of Child Health

$S$ P Devane

P J Milla

Correspondence to:

Ms B S Sokel,

Department of

Psychological Medicine

Hospital for Sick Children, Great Ormond Street,

London WCIN 3JH.

(Arch Dis Child 1990;65:627-7) imaging, skin prick tests, and urine metabolic studies were normal. Administration of metoclopramide, domperidone, and prochlorperazine was not beneficial. Continuous nasojejunal feeding reversed the weight loss and reduced the vomiting.

Individual and family interviews disclosed that the boy was bullied at school. The vomiting led to school avoidance and to increased parental attention. Observation in the hospital ward showed that he made friendships with adults rather than children, suggesting difficulty with peer relationships, and that he chose pursuits such as cooking, dressing up, and doll playing, suggesting immaturity, over closeness to his mother, and over protectiveness within the family.

The vomiting was explained to the patient and to his family as a reflex habit and he was told that he would be helped to control it. He accepted the offer of being taught a self hypnosis technique consisting of relaxation, guided imagery, and suggestions of symptom relief to help control the vomiting. The guided imagery incorporated his enjoyment of cooking and love of animals. It described descending a flight of stairs slowly, becoming more relaxed with each step, and entering a 'special garden'. Here he chose the ingredients for a cake with antiemetic powers, and baked it with the help of woodland animals. Suggestions were made that ' . . y you feel your tummy becoming relaxed and comfortable so that when you eat . . you do not vomit'. The posthypnotic suggestion that the antiemetic action would persist was made. This sequence was recorded on an audio cassette tape.

He was instructed to listen to the tape on his portable personal audio cassette player before meals. A star chart token reinforcement programme was introduced, rewarding him for lengthening periods of postprandial food retention. Privileges and treats were made contingent upon success. Parents and ward staff were instructed to avoid reinforcing the vomiting through attention. Individual counselling sessions to explore his concerns and to teach him stress management techniques were conducted.

Onset of symptomatic relief was rapid and progress was gradual but steady. After three days, he retained food for 20 minutes, after 11 days he retained food until after a subsequent meal, and after 17 days he had stopped vomiting. Over 17 days, the nasojejunal feeding was gradually withdrawn. A relapse occurred on the 19th day, concurrent with a chicken pox infection, but recovery was rapid and vomiting had 
stopped completely again by the 26th day. He stopped using hypnotherapy after four weeks and he remains completely free of vomiting 12 months later. He has been seen for follow up, with his family, by his local child psychiatrist on three occasions.

\section{Discussion}

Self hypnotherapy using guided imagery was used as the primary treatment in this 9 year old boy. This exploited the high imaginative skills of children, which peak at the age of 9 years, and the related hypnotic ability. ${ }^{6}$ The imagery material was tailored to the child's own interests and age level, and he was involved interactively in the recording of the material. This involvement captured his interest and motivation, and encouraged an expectation of coping and control. It gave him a means of getting better 'with honour'. ${ }^{4}$

The use of a personal audio system allowed him to use the treatment in a private and personal manner, increasing the perception of his own control over his symptoms and treatment and separating him from family influences.

Behavioural techniques of contingency management were used as a secondary treat- ment. These techniques encouraged his parents to focus on his efforts to become well, reducing inappropriate parental attention and school avoidance, which are common patterns of behaviour observed in families of children with psychosomatic disorders. ${ }^{4}$ Local follow up over the following months allowed these issues to be addressed and may have been helpful in avoiding later relapses.

Self hypnosis has few or no side effects. It is more acceptable to patients and staff than the use of aversive stimuli. Its successful use in this case suggests that further studies are justified.

1 Schaefer CE, Millman HL, Levine GF. Therapies for psychoJossey-Bass, 1979.

2 Nakanishi DA, Anderson DR. Behavioral treatment of psychogenic vomiting among children - a review and case $17-20$.

3 Kohen D, Olness K, Colwell S, Heimel A. The use of ment of 505 pediatric behavioral encounters. Developmental and Behavioural Pediatrics 1984;5:21-5.

4 Bentovim A. Less orthodox approaches to behaviour problems in children. Arch Dis Child 1985;60:1198-201.

5 Zeltzer LK, Kellerman J, Ellenberg L, Dash J. Hypnosis for reduction of vomiting associated with chemotherapy and disease in adolescents with cancer. $\mathcal{F}$ Adolesc Health Care 1983;4:77-84.

6 Gardner GG, Olness K. Hypnosis and hypnotherapy with children. New York: Grune and Stratton, 1981. somatic disorders in children. San Francisco and London: example. I Psychosoc Nurs Ment Health Serv 1982;20. relaxation-mental imagery (self-hypnosis) in the manage-

\section{Glucose polymer regimens and hypernatraemia}

\author{
I G Verber, M Bain
}$$
3
$$

\begin{abstract}
A 3 year old boy who had glutaric aciduria diagnosed at 22 months of age was admitted with a history of lethargy, vomiting, and fever. He had been receiving glucose polymers as part of his dietary management. He was severely hypernatraemic, but after resuscitation and rehydration made a good recovery. The possible aetiology of his hypernatraemia is discussed.
\end{abstract}

Glucose polymers are being increasingly used as dietary supplements for children. They are particularly useful in the mangement of metabolic decompensation in disorders of amino acid metabolism such as glutaric aciduria. This is an inherited disorder of lysine and tryptophan metabolism caused by glutaryl coenzyme A dehydrogenase deficiency in which slowly progressive neurological deterioration is punctuated by episodes of metabolic decompensation that present with lethargy, vomiting, a reduced level of consciousness, and hypoglycaemia. The mainstay of treatment is a diet low in lysine and tryptophan; this reduces excretion of the metabolite but has a variable effect on the progress of the neurological deterioration. Episodes of metabolic decompensation are managed by stopping the dietary protein intake and giving glucose (usually in the form of a glucose polymer) to prevent hypoglycaemia and provide an alternative source of energy.

We report the case of a child with glutaric aciduria who was given this oral emergency regimen during an attack of gastroenteritis and subsequently developed severe hypernatraemia dehydration.

\section{Case report}

A 3 year old boy was admitted with a three day history of lethargy, vomiting, and fever. Glutaric aciduria had been diagnosed at the age of 22 months. He was on a diet in which his intake of tryptophan and lysine was carefully controlled. In response to these symptoms his parents had stopped his dietary protein intake and given him feeds of an oral glucose polymer (Maxijul, Scientific Hospital Supplies). They made this feed up to $20 \%$ strength (w/v) and to each $200 \mathrm{ml}$ they added a sachet of glucose electrolyte mixture (Dioralyte, Rorer); this provided an additional $35 \mathrm{mmol} / \mathrm{l}$ sodium and $40 \mathrm{~g} / 1$ glucose. He was given this feed every four hours at first, but as he became more dehydrated this was increased so that in the 24 hours before admission he had had nine feeds giving approximately $150 \mathrm{ml} / \mathrm{kg} /$ day. He had no additional free water. On the day of admission he had become extremely drowsy and developed profuse watery diarrhoea. 